Voix et Images

volxetimages

\title{
Volkswagen Blues : traverser les identités
}

\section{Pierre L’Hérault}

Volume 15, numéro 1 (43), automne 1989

Jacques Poulin

URI : https://id.erudit.org/iderudit/200813ar

DOI : https://doi.org/10.7202/200813ar

Aller au sommaire du numéro

\section{Éditeur(s)}

Université du Québec à Montréal

\section{ISSN}

0318-9201 (imprimé)

1705-933X (numérique)

Découvrir la revue

\section{Citer cet article}

L’Hérault, P. (1989). Volkswagen Blues : traverser les identités. Voix et Images, 15(1), 28-42. https://doi.org/10.7202/200813ar d'utilisation que vous pouvez consulter en ligne.

https://apropos.erudit.org/fr/usagers/politique-dutilisation/ 


\section{Volkswagen Blues : traverser les identités}

\section{par Pierre L'Hérault, université Concordia}

\section{Selon Pierre Nepveu, le cas Poulin demeure on ne peut plus éclairant pour} la culture québécoise actuelle, le vieux Volks de son personnage Jack Waterman ${ }^{1}$ pouvant être conçu comme une métaphore même de la nouvelle culture québécoise: indéterminée, voyageuse, en dérive, mais «recueillante» 2. Rapprochée de l'interprétation de Jean Levasseur qui voit tous les romans de Jacques Poulin caractérisés par le mal de vivre dans une société moderne qui rejette totalement le rêve, mal de vivre qui, dans Volkswagen Blues, se transforme en virtuelle quête d'identité ${ }^{3}$, la proposition de Nepveu, d'une part, montre ce que ce dernier roman ajoute aux précédents, en quoi il les explicite et, d'autre part, indique à quel point il apparaît pertinent à l'actualité québécoise, si l'on veut bien entendre par là ce questionnement du discours identitaire et culturel, de plus en plus présent et explicite depuis le début des années 80. Car, traitant du rapport à l'Amérique, à la fois perte et possession, oubli et mémoire, passé et présent, imaginaire et réel, il s'inscrit dans l'exploration d'une culture et d'une identité qui ne peuvent plus être vues comme pures, mais nécessairement métisses, non contraintes en des frontières étanches, mais en quelque sorte transfrontalières, lieux de croisement, de confluence. La complexité et l'ambivalence des rapports de Jack et Pitsémine avec cette Amérique, connue et inconnue, familière et lointaine, douce et violente, aimée et haïe, appellent le recours au mien-étranger et à l'étrangermien ${ }^{4}$ de Mikhail Bakhtine, à la non-identité et au non identifiable ${ }^{5}$ d'André Belleau, concepts appropriés à la lecture d'une œuvre qui en illustre l'efficacité opératoire. Pour en arriver à déterminer la position de Volkswagen Blues dans le texte québécois et le discours identitaire, seront examinées d'abord les figures de la quête et du voyage, ensuite celle du métissage appliqué aux personnages et, finalement, quelques références littéraires.

1 Jacques Poulin, Volkswagen Blues, Montréal, Québec/Amérique, 1984, 290 p. (Littérature d'Amérique). Dorénavant, les références à ce livre ne se feront que par l'indication de la page.

2 Pierre Nepveu, l'Eccologie du réel. Mort et naissance de la littérature québécoise contemporaine, Montréal, Boréal, 1988, p. 216.

3 Jean Levasseur, «L'ambiguitté temporelle dans Jimmy de Jacques Poulin», l'Espace-temps dans la littérature, textes réunis et présentés par Maïr Verthuy, les Cahiers de l'APFUCC, série III, no 2, 1989, p. 49.

4 Mikhail Bakhtine, «Les carnets. 1970-1971», Esthétique de la création verbale, Paris, Gallimard, 1979, p. 365.

5 André Belleau, Surprendre les voix, Montréal, Boréal, 1986, p. 104. 


\section{De la *finalité» de la quête à la «matérialité» du voyage}

La distanciation du discours commun, consacré et normatif se manifeste d'abord dans l'utilisation parodique de la figure de la quête. Si en effet, la figure de la quête désigne à la fois la tension entre le sujet et l' objet de valeur visé, et le déplacement de celui-là vers celui-ci ${ }^{6}$, il est évident que la quête est ici un échec, puisque l'aspect terminatif ${ }^{7} n$ 'est pas réalisé. Le roman est compris entre les deux phrases suivantes de Jack: Je cherche mon frère [...] (p. 11) et [...] l'idée qu'il vaut mieux ne pas revoir mon frère... (p. 289). Si donc la première phrase indique que toute l'action est motivée par la recherche de Théo, la deuxième enregistre qu'il s'agissait là d'une fausse motivation, d'une sorte de trompe-l'œil: [...] maintenant je me demande si j' aimais vraiment Théo. Peutêtre que j'aimais seulement l'image que je m'étais faite de lui (p. 289). Le personnage ne considère pas pour autant son aventure comme un échec. C'est là une donnée fondamentale que Gilles Marcotte a formulée de la façon suivante: On [Jack d'abord] comprend que ce n'était pas, en vérité, pour Théo que Jack avait entrepris le voyage. C'était pour le voyage. ${ }^{8}$ On serait passé du régime de la «finalité», de la «linéarité», de l'«intentionnalité» de la quête à celui de la «matérialité», de la "discontinuité», de l'«inattendu» du voyage. Comment douter que cet ordre ne soit préféré à l'autre quand on sait que c'est grâce au «hasard» que Jack a rencontré Pitsémine, sa compagne de voyage, quand on voit avec quelle facilité et quelle rapidité il se résigne à ne plus revoir son frère pour qui il a traversé le continent, quand on constate sa disposition à tirer parti de l'imprévu et même à le favoriser! Implicite au schéma narratif dont elle fait une contrefaçon du modèle narratif occidental, l'intention parodique est explicitée par la référence à la figure matricielle de la quête, la recherche du Saint-Graal (p. 99). Il s'agit moins en fait de la référence elle-même, en quelque sorte obligée, que de la manière dont elle est amenée. On n'eut pas été surpris de la voir introduite par Waterman qui l'aurait extraite d'un savoir littéraire légitimement acquis ou cautionné par son statut d'écrivain. Au lieu de quoi c'est une femme métisse, Pitsémine/la Grande Sauterelle, qui la glisse dans le texte. Et encore! pas à partir d'une édition critique, ni même d'une anthologie ou d'une histoire littéraire consultée dans une bibliothèque, mais à partir de l'Encyclopédie de la Jeunesse, lue dans la roulotte de son père, non pas au cours d'une docte conversation, mais à cause d'un panneau de signalisation. entre Chatham et Windsor, qui indiquait le comté de Merlin. Cette façon non conventionnelle, triviale, de remonter au roi Arthur, à sire Lancelot du Lac, d'évoquer la figure de ce jeune et noble chevalier à côté d' un cheval blanc [...] illuminé par la vision du Saint-Graal équivaut à un détournement de sens, à une perte de la finalité d'une image qui ne sert plus à se réclamer d'une tradition

6 Algirdas Julien Greimas et Joseph Courtés, Sémiotique. Dictionnaire raisonné de la théorie du langage, Paris, Hachette Université, 1979.

7 Ibid.

8 Gilles Marcotte, «Histoires de zouaves», Études françaises, vol. XXI, no 3, hiver 1985-1986, p. 7-17. Reproduit dans Littérature et circonstances, Montréal, 1'Hexagone, 1989, p. 301 (Essais littéraires). 
littéraire et culturelle qu'elle condense et à laquelle on a dûment été initié, mais à signaler un lieu (le comté de Merlin) et à faire émerger une expérience d'enfance (lorsqu'elle était petite). Ce processus de désacralisation indique qu'on n'a pas affaire à un discours autoritaire et dogmatique, mais à un discours proposé sous le mode de la coopération et non de la violence ${ }^{9}$.

Nous ne sommes pas en présence de personnages unidimensionnels, fixés par une définition préexistante, pour qui l'accomplissement viendrait uniquement de l'atteinte d'un but ou de l'actualisation de virtualités originelles, mais de personnages saisis comme récepteurs actifs, sensibles et ouverts à la modification, plus attentifs à l'imprévu des détours des petites routes qu'à l'uniformité de l'autoroute. Pitsémine résume cette disposition à travers une phrase de Daniel Boone que Jack approuve par son silence: Je me sens parfois comme une feuille sur un torrent. Elle peut tournoyer, tourbillonner et se retourner, mais elle va toujours vers l'avant. (p. 289) La dialectique de la mémoire et du présent, sur laquelle se structure l'univers romanesque de Volkswagen Blues, se retrouve définie comme une négociation, parfois serrée, entre la mémoire et la conscience du présent, entre une identité imposée et une identité complètement ouverte. Je retiens le mot «négociation», car Poulin semble ne vouloir éliminer ni l'un ni l'autre des termes, mais les considérer comme deux composantes indissociables. Dans Volkswagen Blues, les personnages ne rejoignent jamais uniquement l'Amérique physique, ni uniquement l'Amérique de la mémoire. L'une interférant inévitablement sur l'autre, les personnages n'échappent ni à la mémoire, ni au présent, ne peuvent atteindre l'une sans passer par l'autre. Ils ne peuvent s'isoler ni dans l'une, ni dans l'autre. Leurs rapports mêmes sont fortement tributaires de leur mémoire particulière de l'Amérique. S'ils habitent un même présent, parcourent un même continent, leur façon de s'y rejoindre est conditionnée par leurs rapports imaginaires à cet espace présent, objectivement le même, et pourtant imaginairement différent. De cela rendent compte les cartes de l'Amérique du Nord que consultent Pitsémine et Jack au cours de leur voyage.

Les cartes superposent trois Amériques bien distinctes. Sur celle de l'Amérique du Nord du $18^{e}$ s., possession de la France, Jack contemple une possession perdue, espace des exploits de ses ancêtres français et canadiens, «découvreurs», explorateurs, colonisateurs, coureurs de bois, qui la marquèrent, y établissant des routes, des postes, des habitations, des fondations, etc. De leur passage il ne reste plus guère, en dehors du Québec, que des traces. L'imparfait répété insiste sur ce qui a été, mais n'est plus:

Ils regardèrent en particulier une très grande et très belle carte géographique de l'Amérique du Nord où l'on pouvait voir l'immense territoire qui appartenait à la France au milieu du $18^{e}$ siècle, un

9 Umberto Eco, Lector in fabula. Le rôle du lecteur ou la coopération interprétative dans les textes narratifs, traduit de l'italien par Myriem Bouzaher, Paris, Grasset, 1985, p. 71. 
territoire qui s'étendait des régions arctiques au golfe du Mexique et qui, vers l'ouest, atteignait même les montagnes Rocheuses: c'était incroyable et très émouvant à regarder. (p. 20)

Émouvant pour Jack surtout! Car, suit immédiatement, dans le même paragraphe une autre carte géographique, tout aussi impressionnante, qui montrait une Amérique du Nord avant l' arrivée des Blancs: celle de Pitsémine. Deux Amériques sont ainsi isolées, avant d'être irréductiblement opposées par le rapport qu'entretient avec elles chacun des personnages. Les choses se présentent dans l'ordre suivant. D'abord, Jack en est exclu, de l'extérieur, par le avant l'arrivée des Blancs et, de l'intérieur, par sa méconnaissance (son absence de mémoire) d'une grande quantité de noms dont il n'avait jamais entendu parler de toute sa vie. Ensuite, Pitsémine y est rattachée en exclusivité par l'émotion qu'elle manifeste en la parcourant et que ne peut partager Jack: La fille s'attardait longuement devant la deuxième carte. Ses yeux étaient brillants et humides [...]. Cette dernière remarque renforce l'impénétrabilité des deux Amériques en établissant clairement que l'émotion ressentie devant la première carte, qu'on pouvait, jusque-là, mettre au compte des deux personnages, n'était le fait que de Jack. Chacun est donc isolé dans sa propre mémoire: [...] et Jack comprit qu'il valait mieux la laisser toute seule un moment. Il revint dans le hall. Par contre, Jack et Pitsémine s'entendent très bien sur la lecture des «cartes routières», en quantité invraisemblable (p. 26) dans le minibus. Jack ne trouve rien à redire au tracé que fait Pitsémine de la route qui semblait être la plus normale pour aller de Gaspé à Saint Louis. La suite du voyage montre d'ailleurs qu'ils trouvent dans l'Amérique présente un véritable terrain d'entente. Les nombreux détails concrets du voyage, minutieusement notés (tracés des parcours, relais, manière de conduire, vie commune dans le minibus, etc.) l'indiquent. Du point de vue des rapports entre les personnages, on peut donc distinguer très clairement le passé qui les isole du présent qui les unit. On aura noté une autre distinction: alors que le passé est représenté comme statique («cartes anciennes» contemplées dans un «musée»), le présent est représenté comme en déplacement («cartes routières» consultées «sur la route»). Il faut s'arrêter à cette double distinction, car elle fonde la dialectique «mien-étranger»/«étranger-mien» sur laquelle sont construits les personnages.

\section{Le métissage des personnages}

Avant de quitter le Musée de Gaspé et de replier les cartes routières, nuançons ce qui a été dit au sujet de l'impénétrabilité réciproque des Amériques française et amérindienne. S'il est vrai, dans le passage étudié, que les protagonistes se trouvent isolés dans deux univers qui semblent devoir rester étrangers, par contre, ailleurs, un ailleurs qui, dans le roman, n'est souvent qu'à côté, les deux univers se retrouvent dans le rapport inclusif, mais non-fusionnel, du «mien-étranger» et de l' «étranger-mien», plutôt que dans le rapport exclusif du «mien» et de l'«étranger». Ainsi, Pitsémine s'adresse en ces termes à Jack, connotant son vouvoiement d'un sens collectif qui ne trompe pas sur la distance qu'elle met entre elle et lui, ni sur la nature et les causes de cette distance: 
Quand vous ${ }^{10}$ parlez des découvreurs et des explorateurs de l'Amérique... Moi, je n'ai rien en commun avec les gens qui sont venus chercher de l'or et des épices et un passage vers l'Orient. Je suis du côté de ceux qui se sont fait voler leurs terres et leur façon de vivre. [...] On est arrivés par l'Ouest et vous êtes arrivés par l'Est. Il y a 7000 kilomètres qui nous séparent! (p. 28)

Le narrateur ajoute: Tout à coup elle se mit à rire, avant de rendre la parole à Pitsémine qui explicitera le sens de son rire et redonnera au vous sa dimension individuelle: Excusez-moi [...]. J'étais en train de me prendre au sérieux!... $D^{\prime}$ ailleurs, je ne suis pas une vraie Indienne. Mon père est un Blanc. Je suis une Métisse. Les propos et le rire de Pitsémine valident la théorie de Bakhtine, selon laquelle la «monotonalité» (le sérieux unilatéral) est associée au dogmatisme, à l'autoritaire, à la violence, à la mort et la «pluritonalité» (sérieux-rire), à l'ouverture, à l'égalité, à la liberté, à la vie ${ }^{11}$. Pitsémine doit convenir en effet qu'elle ne peut «sérieusement» tenir un discours monologique, non pas en vertu d'une position idéologique ou affective, mais en vertu de la réalité, de la vie. Agir autrement serait, pour elle, se nier, se détruire. Sa condition de Métisse ne lui laisse pas le choix de son identité hybride, de son appartenance à deux mémoires, sous certains aspects, non seulement opposées, mais irréconciliables.

Les choses ne sont pas aussi tranchées pour Jack qui n'est pas un Métis ${ }^{12}$. Il n'en reste pas moins que l'amérindianité peut lui être attribuée comme un «étranger-mien». Retenons, pour l'instant, à titre d'illustrations, deux passages du chapitre VI. Dans le premier, Jack évoque ses jeux d'enfant, entre autres les interminables batailles opposant les Blancs et les Indiens du voisinage (p. 64). L'expression est, volontairement sans doute, ambiguë. Littéralement, on parle de «vrais» Blancs et de «vrais» Indiens. Le contexte cependant laisse croire que ce sont tous des écoliers «blancs» divisés en deux bandes, dont l'une joue les Indiens. L'histoire d'Étienne Brûlé, qui entrecoupe le récit de l'enfance, tendrait à le confirmer. Dans le deuxième passage, en effet, Théo cherche à communiquer à ses camarades sa passion pour Étienne Brûlé, son héros, et, par voie d'identification, celui de Jack. Pourquoi le prend-il pour héros? Laissons à Jack le soin de le dire: Il racontait [...] comment Étienne Brûlé, arrivant en Nouvelle-France avec Champlain, avait obtenu la permission de vivre avec les Indiens pour apprendre leur langue et comment il vivait à la manière des

10 C'est moi qui souligne.

11 Mikhail Bakhtine, op. cit., p. 354.

12 Pourtant, lecteur invétéré de Ferron, je ne puis m'empêcher de considérer cette possibilité à cause du «Pape Poulin» (la Chaise du maréchal ferrant), beauceron comme notre auteur, qui habite les «Profonds» de la Beauce, région, selon Ferron, d'échanges entre Amérindiens et Canadiens, d'où l'on pouvait du reste, puisqu'elle était située tout près de la frontière américaine, hardiment pisser dans les EtatsUnis (p. 74). L'auteur me pardonnera ce rapprochement tout à fait extra-textuel qui se donne des airs de sérieux, en jouant d'une façon hasardeuse, pour ne pas dire interdite sur les patronymes et les prénoms. Mais on lit avec tout ce que l'on est. Aussi bien l'avouer! 
Indiens, qui l'avaient adopté comme l'un des leurs [...]. (p. 66) La dernière expression, comme l' un des leurs, identifie la qualité qui, aux yeux de la «bande à Théo», faisait d'Étienne Brûlé un héros: son identification avec les Indiens, sa presque indianité (vivre avec, apprendre leur langue, vivait à la manière des Indiens, qui l'avaient adopté), fait qu'il puisse pratiquement être assimilé aux Amérindiens. Voilà donc de quelle façon Jack revendique comme sienne l'identité amérindienne. Mais ce type d'appropriation facile conduit à la négation de l'Amérindien, qu'elle s'exprime à travers le mythe de «l'Amérindien sanguinaire» (les jeux des enfants) ou à travers celui du «sang amérindien dans les veines» (l'histoire d'Étienne Brûlé). Jack en prendra cruellement conscience lorsqu'il se fera dire, à la bibliothèque de Toronto, qu'Étienne Brûlé était un bum (p. 71). Aussi, est-ce avec de l'agressivité dans sa voix et aussi un mélange de tristesse et de lassitude (p. 75), qu'il demandera à Pitsémine, qui usera de toutes les précautions voulues, de confirmer le jugement précité.

L'expression «démarcation conciliante» et le principe Les démarcations ne perdent rien à être fortement marquées, mais il faut qu'elles soient conciliantes ${ }^{13}$ que Bakhtine utilise à propos de la science trouveraient ici une autre application. Car si le jeu des rapports entre Pitsémine et Jack postule des personnages nécessairement métisses, cela n'est pas le résultat d'un processus de fusion, de réduction de l'altérité. La dynamique des rapports des personnages repose au contraire sur des démarcations [...] fortement marquées, mais [...] conciliantes. À commencer bien sûr par leur statut romanesque qui en fait des personnages distincts, autonomes dans leurs paroles et leurs gestes. Ce ne sont pas des demipersonnages, des voix du narrateur ou de l'auteur, mais des personnages entiers, possédant une biographie propre, une culture propre, des habitudes particulières. Pour le lecteur et la lectrice, Pitsémine n'est pas une vision de Jack. Elle monte vraiment dans son Volks et fait bel et bien avec lui le voyage de Gaspé à San Francisco, comme c'est écrit. La «démarcation» des personnages est une donnée fondamentale du roman, dont rend compte ce segment de dialogue: - En général, j'aime mieux être toute seule. - Moi aussi. (p. 290) Le dialogue cependant ne s'arrête pas là et contient une autre donnée tout aussi factuelle, et tout aussi nécessaire, bien que contradictoire, à l'existence de ce roman: - Et pourtant on est restés ensemble tout l'été. - On ne peut pas toujours être logique! (p. 290) Tout le récit ne repose-t-il pas sur l'acceptation par le lecteur et la lectrice de cette faille de la logique, qui permet les «démarcations conciliantes» et que l'auteur sait rendre acceptable? Comment autrement lire Volkswagen Blues sans voir dans certains passages de grossières invraisemblances (le fait, par exemple, que Jack n'entreprenne la recherche de son frère que quinze ans après la réception de sa demière carte postale)?

Les rapports de Jack et de Pitsémine sont un jeu constant de déplacements à l'intérieur d'un champ identitaire d'où le monologique est exclu. Jack n'est jamais Pitsémine; pourtant il l'est. De même Pitsémine n'est jamais Jack; pourtant elle l'est de quelque façon. Leurs rapports oscillent entre deux

13 Mikhain Bakhtine, op. cit., p. 356. 
extrêmes, allant de leurs réciproques retranchements mutiques à ces moments où ils se devinent, se partagent corps et âme, en passant par ces ententes et discordes momentanées qui composent le quotidien. Mais Jack n'est-il que Jack et Pitsémine que Pitsémine? Jack est aussi Jacques; Pitsémine est aussi la Grande Sauterelle. Dans ce jeu de rapprochement/éloignement, de dédoublement, le personnage se déplace, non seulement par rapport à l'autre, mais aussi par rapport à lui-même, dans un mouvement de décentrement qui va de l'unique au multiple. Parler de tous les doubles de Jack et de Pitsémine 14 ne suffit pas à rendre compte du phénomène; il faut de plus préciser qu'il y a une force d'attraction qui agit dans le récit pour lui donner une direction: celle du multiple, représentée par Pitsémine/la Grande Sauterelle, la Métisse. Parti à la recherche d'un frère qu'il avait façonné à son image (p. 289), Jack rencontre l'autre, femme et métisse, Pitsémine, qui, s'amusant à jouer la psychologue, l'amène à prendre conscience du narcissisme de sa quête et à s'interroger sur «les rapports entre les gens». Les derniers mots du roman évoquent cet espace de l'altérité auquel accède Jack: [...] il souriait malgré tout à la pensée qu'il y avait, quelque part dans l'immensité de l'Amérique, un lieu secret où les dieux des Indiens et les autres dieux étaient rassemblés et tenaient conseil dans le but de veiller sur lui et d'éclairer sa route.

La structuration romanesque met donc ici en place, à partir des cartes géographiques et routières qui figurent leur espace identitaire et culturel, deux personnages dont la conscience identitaire apparaît en quelque sorte poreuse ${ }^{15}$, trouée, délimitée par une frontière plus imaginaire (p. 54) que réelle et étanche. Il y a là une vision de l'identité, comme lieu de déplacements qui déborde évidemment la dimension «québécoise» des personnages. Et pour ne pas être trop incomplet, tout en restant dans les limites de notre propos, il faut ajouter, par exemple, qu'il y a en plus du déplacement de la «francité blanche» de Jack vers Pitsémine, de l'«amérindianité» de Pitsémine vers Jack, un déplacement du masculin de Jack vers Pitsémine et du féminin de Pitsémine vers Jack. Il y a d'ailleurs beaucoup d'humour employé à faire se déplacer l'écrivain-mâle-blanc de sa fonction sacrée d'écrivain à la vaisselle et aux diverses tâches ménagères et la femme-métissefille-de-camionneur-blanc-et-de-femme-de-ménage-amérindienne de la primitivité et de la mécanique aux livres (c'est elle après tout la «cultivée», elle qui en sait le plus!) ${ }^{16}$. En fait, on pourrait définir comme suit ce modèle de déplacement: dans

14 Ils sont trop nombreux pour qu'on cherche ici à en donner la liste, d'autant que, fondés sur le métissage, ils se multiplient presqu'à l'infini...

15 Cette idée de «porosité», rencontrée dans la Québécoite (Montréal, Québec/ Amérique, 1983, 201 p.) de Régine Robin, dans un contexte similaire à celui-ci, mérite d'être suivie, car elle aurait un fondement dans la philosophie baroque, selon Gilles Deleuze (le Pli. Leibniz et le baroque). Or on sait les liens qu'établit Umberto Eco entre le baroque et sa notion d'cuvre ouverte (l'CEuvre ouverte).

16 On pourrait, me semble-t-il, établir un rapport intéressant et valable entre cette situation romanesque et celle de l'Euguélionne (Montréal, la Presse, 1976, 399 p.), de Louky Bersianik. Dans les deux romans, la femme, qui «connaît» et introduit un regard «critique», vient d'ailleurs: chez Poulin, d'une autre race; chez Bersianik, d'une autre planète. 
le récit, un personnage masculin accomplit un parcours qui le mène du pouvoir à l'impouvoir $^{17}$, du centre à la périphérie, à la marge ${ }^{18}$.

\section{Deux écrivains et une écrivaine de la marge québécoise}

Il n'est pas étonnant dès lors qu'apparaissent, parmi les références littéraires les plus importantes, trois auteurs de la marge québécoise: la Québécoised'origine-manitobaine Gabrielle Roy, le Franco-Américain Jack Kerouac et le Judéo-Canadien-américain-d'origine-québécoise Saul Bellow. Cherchons la fonction de ces références en commençant par relever à propos du livre cité de Gabrielle Roy, Fragiles Lumières de la terre, un certain nombre de détails. Le livre a comme sous-titre «Écrits divers 1942-1970»; cherchant à préciser l'«intérêt» que peut présenter la réunion de ces textes, la romancière écrit:

Il se peut qu'ils aident à retracer le chemin parcouru depuis la rue Deschambault via l'Europe jusqu'à Montréal puis Québec où je suis à présent fixée, chemin que je n'ai guère vu moi-même - voit-on seulement son chemin au fur et à mesure que l'on avance dans sa vie et dans son ouvre? (p. 9) 19

Enfin, Roy retient surtout [...] les reportages des années quarante sur les Peuples du Canada, sortes de portraits de communautés ethniques alors assez bien préservées, que j'eus tant de joie à découvrir et à faire connaître ${ }^{20}$. Ces sept reportages constituent la plus grande partie du livre ${ }^{21}$, placé par son

17 Le sens que Madeleine Gagnon donne à ce mot, qu'elle reprend à Blanchot et Artaud, est tout à fait approprié ici («Discours de réception à l'Académie canadienne-française», Toute écriture est amour. Autographie 2, textes réunis et présentés par Jeanne Maranda et Maïr Verthuy, Montréal, VLB éditeur, 1989, p. 178-183).

18 Toute la question du «féminin» dans Volkswagen Blues constitue à elle seule un sujet d'étude. Son examen ne saurait donc en être entrepris ici. Je rappellerai seulement qu'elle n'est pas nouvelle dans l'œuvre de Poulin, mais représente une constante et que dans Volkswagen Blues elle se combine avec l'amérindianité. La femme (Pitsémine) est métisse, mais il est évident dans l'économie du roman que dans cette conjonction d'identités, c'est l'amérindianité qui prédomine. Cela n'est pas une interprétation fantaisiste, mais repose sur les données romanesques suivantes: Pitsémine est amérindienne par sa mère qui est la seule des deux parents à exister comme personnage dans le roman. Elle joue même un rôle relativement important en identifiant sur la carte postale de Théo l'écriture de Jacques Cartier (p. 18); c'est donc elle qui met sur la «piste».

19 «Présentation», Fragiles Lumières de la terre, Montréal, Stanké, 1982, p. 9. En italiques dans le texte.

20 Ibid. En italiques dans le texte.

21 Ils composent, avec un texte intitule «Le Manitoba» (p. 101-120) et deux textes regroupés sous le titre «Paysages de France» (p. 123-140), la première («I-Reportages) des trois sections et de beaucoup la plus importante, occupant les pages 13 à 140 , alors que les deux autres occupent respectivement les pages 143 à 197 («IISouvenirs») et 199 à 233 («III-“TERRE DES HOMMES": le thème raconté»). Si l'on note enfin, qu'à l'exception de celui de la troisième section, tous les textes réunis dans Fragiles Lumières de la terre parlent d'une réalité autre que québécoise, on comprendra l'importance de cette référence, compte tenu de la perspective où je me place pour lire le récit de Poulin. 
auteure sous le signe de la diversité, de l'imprévisible, de l'errance et de la pluralité ethnique ${ }^{22}$. Une toute petite phrase condense l'attrait qu'excerce le livre de Roy sur Waterman: Et il fut ainsi renvoyé à lui-même et d sa propre écriture (p. 47). Connaissant la logique des rapports qui unissent Jack et Pitsémine, on ne s'étonnera pas qu'à la question: Aimez-vous le livre de Gabrielle Roy? (p. 46) elle réponde: Je l'aime beaucoup. Quant à la référence à Jack Kerouac, dont le titre On The Road apparait dans la liste des affaires de Théo (p. 74) dénombrées lors de son interrogatoire policier à Toronto, elle ne surprend pas quand on nous a déjà dit à son sujet: $\mathrm{Ce}$ qu'il aimait, c'étaient les voyages, les autos. Il faisait des petites jobs et quand il avait un peu d'argent, il partait en voyage (p. 13), ni quand on note que Waterman et Kerouac ont le même prénom. Mais, peut-être, pour en comprendre vraiment la portée, faut-il retourner au premier roman de Kerouac, The Town and the City, dont Maurice Poteet souligne le caractère hybride en parlant d'une auvre obscure pour de nombreux québécois, au statut relativement incertain pour le lecteur américain, de sa vision à la fois «marginale» et tout à fait américaine dans ses antécédents historiques [l'émigration] ${ }^{23}$. Voilà qui rejoint, me semble-t-il, la démarche de Jack Waterman cherchant dans l'écriture et le voyage à explorer le "Grand Rêve de l'Amérique» qui ne serait peut-être finalement que l'image d'un pays idéal en compensation du paradis terrestre perdu et introuvable (p. 101). Gardons-nous cependant de ne voir, chez Kerouac comme chez Poulin, dans ce «pays idéal» et ce "paradis terrestre» perdu et introuvable que des lieux philosophes ou mystiques, détachés de l'histoire et de ses contingences.

La référence à Saul Bellow, Prix Nobel de littérature en 1976, est plus inattendue peut-être dans un texte québécois et mérite une attention particulière. Puisque c'est d'après la notice biographique du Petit Robert 2 que Bellow est présenté (p. 111), citons-la dans sa presque entièreté:

BELLOW (Saul). O Romancier américain (Lachine, Québec, 1915 -). Issu d' une famille d' immigrants israélites, il campe des exilés errants, humiliés, presque tous marqués par les problèmes de la minorité judéo-américaine [...]. Plus optimiste est son grand roman picaresque Les Aventures d'Augie March (1953) qui se termine sur ces mots: «Je suis une sorte de Colomb pour tous ceux qui sont à portée de la

22 Jack Waterman eût sans doute été fort intéressé par l'autobiographie de Gabrielle Roy, la Détresse et l'enchantement, publiée la même année que Volkswagen Blues, qui développe abondamment ces thèmes. Il eut sans doute été fort curieux d'y apprendre que c'est en Angleterre qu'il devint clair pour Gabrielle Roy qu'elle écrirait en français et d'y noter les rapprochements faits, au niveau des images en tout cas, entre l'aventure des pionniers de la piste de l'Oregon et celle des pionniers de l'Ouest canadien. Pour ma part, j'ai abordé la question de l'errance et du féminisme chez Gabrielle Roy dans «Le récit maternel acadien, principe organisateur du temps et de l'espace ou Bonheur d'occasion lu à la lumière de la Détresse et l'enchantement», l'Espace-temps dans la littérature, textes réunis et présentés par Maïr Verthuy, les Cahiers de l'APFUCC, série III, no 2, 1989, p. 53-75.

23 Maurice Poteet, "Avant la route, le village», Voix \& images, vol. XIII, no 3, printemps 1988, p. 388 et 396. 
main et je crois fermement qu'on peut les rejoindre dans cette terra incognita immédiate qui s'étend devant chaque regard...24

Bellow, d'après cette présentation, cumule les identités, les cultures, les nationalités: romancier américain, Québécois de naissance, appartenant à la tradition juive, se reconnaissant dans le génois Colomb qui put voguer à la "découverte» grâce à l'appui de la reine de Castille. Non seulement est-il le nœud vivant de plusieurs traditions, mais encore, par et dans son œuvre (c'est ce qu'en dégage le Petit Robert 2), la conscience de cette jonction. Son lieu de naissance lui-même, un beau cas de symbolique ironique, participe de cette confusion identitaire. Quel lieu d'origine pouvait mieux lui convenir que celui-ci dont le toponyme enregistre l'une des plus énormes erreurs de localisation qui aient été commises, en vertu de laquelle des hommes et des femmes se verront attribuer une fausse identité qui les poursuivra, dissociant déjà, fortuitement il est vrai, territoire et culture.

C'est à Saul Bellow que l'auteur confie l'un des propos les plus importants du roman. Il s'agit du dialogue de la fin du chapitre X (p. 109-110), entre Bellow et Pitsémine:

- What do you think of Chicago? demanda-t-il. She's been a wicked city in her time, but...

- Wicked? fit la Grande Sauterelle.

- I mean rough. Now the violence is still here but it's mixed with business and culture. This is the city that gave birth to the Encyclopaedia Britannica, Zenith TV's, Wrigley's gum, Quaker Oats, and McDonald's hamburgers. But take a walk downtown and there are sculptures and paintings by Picasso and Calder and Chagall... Strange city... I don't know if I like her or not. But I think she's in my blood.

Le propos de Bellow est double. Il décrit la ville de Chicago comme le lieu de l'hétérogène où se côtoient les choses les plus diverses, les plus belles comme les plus laides (violence, affaires, culture, etc.). Il ne s'agit pas de décider si cela est bien ou mal; c'est de l'ordre des faits. Aussi se refuse-t-il à porter un jugement de valeur sur cette situation: I don't know if I like her or not. Cependant, ce dont il est sûr, c'est que cette mixité lui convient. Il l'a dans la peau, cette ville: $[. .$. she's in my blood. Le statut particulier (non seulement d'auteur cité, mais d'auteur devenu personnage) accordé à Bellow surprend, non pas à cause de ses propos tout à fait convergents avec ce qui a été dégagé jusqu'à maintenant du roman de Poulin, mais parce que ce porte-parole privilégié les tient en anglais et les introduit par des remarques qui pourraient être qualifiées de cyniques, surtout qu'elles sont aussi tenues en anglais. Qu'en est-il?

Les remarques potentiellement cyniques constituent quelques répliques du dialogue qui précède immédiatement le passage déjà cité:

On est en voyage, dit Jack. 
- Just passing through? [demanda Saul Bellow]

- Yes. Oui.

- Like your ancestors Louis Jolliet and Father Jacques Marquette!

- Et Robert Cavelier de La Salle, dit la fille.

Tout le monde se mit à rire.

Ce qui risque ici de blesser n'est pas tant le rappel que les explorateurs français n'ont fait que passer, que l'identification de Jack à ces explorateurs. Bien sûr, on parle ici du voyage. Le Like your ancestors élargit toutefois le commentaire jusqu'à le faire porter sur la condition de Jack, son appartenance à une minorité dépossédée de la plus grande partie du territoire américain marqué par ses ancêtres. Au caractère blessant de ces paroles s'ajouterait leur cynisme du fait que ces cruelles réalités lui sont rappelées en anglais, la langue du conquérant, par l'intermédiaire au surplus d'un écrivain que ce même conquérant s'est approprié. Une telle interprétation ne saurait tenter le lecteur et la lectrice que très provisoirement, soit la durée de deux pages, si du moins l'on se donne la peine, à l'incitation du narrateur (p. 111), d'aller lire dans le Petit Robert 2 la notice biographique de Saul Bellow. Mis au fait des origines et du parcours de Bellow, de la place qu'occupent dans son œuvre les exilés errants, humiliés, presque tous marqués par les problèmes de la minorité judéo-américaine, on devra l'abandonner. Plutôt que du cynisme, peut-être faudra-t-il parler d'«humour juif» s'excercant sur la condition québécoise. Bellow, à travers l'expérience minoritaire, établirait alors un rapport de sympathie, annoncé par l'excitation que manifeste Jack quand il découvre qu'il se trouve en présence de l'écrivain célèbre (p. 107). Ce qui est clair en tout cas, c'est que le narrateur et Jack prennent à leur compte les paroles de Bellow. Poursuivant la lecture du texte, on verra en effet, que, loin de le percevoir comme un être cynique, Jack est sensible au message d'encouragement que décèle Pitsémine dans la phrase de Bellow citée par le Petit Robert: - On a l'impression que Saul Bellow nous dit qu'on est sur la bonne route et qu'il nous souhaite bonne chance. (p. 111)

Cela étant dit, il n'est pas à exclure qu'on puisse voir dans ces propos tenus en anglais par l'«écrivain célèbre», le «Prix Nobel de littérature» dont se réclame le Canada anglais, le rappel d'une situation conflictuelle bien connue, donnée incontournable de la situation minoritaire des francophones d'Amérique, du Québec y compris. Cependant, comme ailleurs dans ce roman, cette situation n'occupe pas tout l'horizon, pas plus qu'elle n'est refoulée. En d'autres mots, elle n'est pas traitée de façon simpliste, c'est-à-dire unilatéralement sérieuse, mais dans le rire. Jack, ou le lecteur, aurait beau s'offusquer des propos de Bellow; il faudrait bien qu'il éclate de rire en se disant que ce fils d'immigrants israélites est pour bien peu dans la Conquête et ses suites! ${ }^{25}$ L'anglais, à la fois

25 Bien sûr, on pourra me rétorquer que justement si Bellow écrit en anglais, c'est une suite de la Conquête. Ce n'est évidemment pas, à la suite de Poulin, sur ce terrain que je me place. Il s'agit ici essentiellement de travailler à dans une perspective synchronique. 
rappel d'une condition minoritaire, mais possibilité d'un ailleurs, fait partie du bagage «mien-étranger». Jack l'écrivain le sait bien qui n'est pas tout à fait étranger au traducteur des Grandes Marées, pour qui l'anglais est l'un des matériaux avec lesquels il travaille. Jack le voyageur sait bien aussi qu'en cette Amérique qu'il parcourt, l'anglais fait partie du bagage identitaire québécois, en un double sens: comme minoritaires en Amérique, on y est contenu; comme majoritaires au Québec, on le contient. L'intrusion de Bellow dans le roman appuie la dialectique du «mien-étranger» qui sous-tend les diverses instances du roman. Tout laisse croire, à cause de l'attitude complice du narrateur, que Bellow (le personnage) saisit très bien la drôlerie, l'incongruïté de la situation qui lui fait tenir le discours du conquérant, lui le Juif errant. Mais ce qu'il importe de savoir, ce n'est pas si le personnage de Bellow trahit l'écrivain Bellow, mais de saisir, dans le dédoublement de Bellow (personnage romanesque et individu réel), la distance ironique que le narrateur prend par rapport à ses préjugés, aux clichés simplificateurs du discours identitaire.

\section{Le minibus ou la maison mobile}

Si l'on cherche, au terme de notre lecture, à préciser de quelle manière Volkswagen Blues est une concrétion esthétique du discours social 26 québécois, il faut, je pense, en premier lieu, dégager sa position non idéologique. Je reprendrais ici volontiers les expressions de Nepveu pour dire que l'œuvre ne travaille pas à nommer, à définir, mais qu'elle se contente d'être «recueillante». Elle est ouverte: elle veut tout accepter, tout accueillir, tout capter. Un seul parti pris: aller au bout du voyage, en empruntant tous les détours qui se présentent. On pourrait comparer le texte à un filet capteur où viendrait se fixer tout ce qui le touche. Il ne discrimine pas; il ne connaît pas la perspective hiérarchisante. Il est plutôt le lieu d'une mise au jour des images et discours sociaux avec lesquels l'individu-récepteur construit une figure de son identité, de sa culture (toujours indéterminée), en déconstruisant ce qui se donne comme l'identité, la culture, dans le sens exact où André Belleau écrit que les écrivains font du neuf avec le discours de leur société ${ }^{27}$. Euvre bigarrée où les citations de textes anciens et «sacrés» côtoient les références à la course automobile, les discussions sur les rapports homme-femme, la mécanique, les questions de vie commune et quotidienne, etc. Jack et Pitsémine ne sont pas des personnages unidimensionnels, mais des personnages à plusieurs voix.

Révélateur plutôt que définisseur, le texte se caractérise encore par sa hantise de ne pas déborder du présent. Il est comparable au compteur qui enregistre infailliblement le kilométrage du minibus. Les nombreuses notations des détails (vie commune, routes empruntées, détours pris, sorties, bruits du moteur, etc.) concernant la matérialité du voyage apparentent le roman à un journal de bord attentif à saisir l'immédiateté des choses. Jack Waterman est bien forcé de reconnaître qu'il n'a rien de l' «écrivain idéal» qu'il imagine: 
Il s'était fait depuis toujours une image de l'écrivain idéal et il était loin de ressembler à ce modèle. Il se rangeait parmi ceux qu'il appelait «l'espèce laborieuse»: patient et obstiné mais dépourvu d'inspiration ou même d'impulsions, il se mettait à l'œuvre tous les jours à la même heure et, grâce à un travail méthodique et opiniâtre, il arrivait à écrire «sa» page quotidienne. (p. 47)

Comme lecteur, l'écrivain Waterman est aussi particulièrement attentif à la facture des phrases, à la matérialité de l'écriture: Il aurait voulu lui dire aussi de ne pas lire trop vite, parce que l'écriture de Gabrielle Roy était très personnelle et que, par exemple, il était toujours intéressant de regarder à quel endroit dans la phrase elle plaçait ses adverbes. (p. 46) L'écriture n'enregistre que le présent: ce qui se fait, ce qui se passe. Si le passé existe, ce n'est donc pas comme refuge, fuite, mais parce qu'il fait partic du présent, comme trace, empreinte, fossile. Ainsi ce texte au présent fait-il exister la mémoire. Ce qui serait moins banal qu'il n'y paraît dans une société où, selon Belleau, les générations se succèdent à tous les cinq ans, où le sens de la continuité apparaît à peu près inexistant, chaque groupe nouveau recommençant tout à neuf pour son propre compte ${ }^{28}$.

Une troisième caractéristique du texte de Poulin pourrait être définie à partir de ce que Belleau appelle la non-identité, le non identifiable auxquels il attribue des valeurs insoupçonnées et trouve des vertus subversives ${ }^{29}$. Nous parlons bien sûr ici de la figure du déplacement, du déportement, du mouvement d'un point fixe (connu) à un ailleurs non déterminé (terra incognita) qui structure Volkswagen Blues, symbolisé le plus visiblement dans le texte par le parcours accompli du Musée de Gaspé (lieu de conservation) à San Francisco (lieu par excellence de changement). Il y a dans cette ex-patriation momentanée et voulue, cette déterritorialisation provisoire et libre, le passage de l'identité à la non-identité, de l'identifiable et de l'identifié au non identifiable et au non identifié. Ce mouvement pourtant n'équivaut pas à l'indifférence, à l'équivalence de toute chose. Il n'y a pas ici réduction du multiple: on ne s'épouse pas comme dans les contes de fées; on ne meurt pas comme dans la tragédie; on ne se suicide pas comme dans le drame shakespearien. On se quitte plutôt banalement, sans drame, emportant quelque chose de l'univers de l'autre: Pitsémine garde le Volks; Jack ramène les dieux de Pitsémine. De même, n'est-on pas devenu autre, au sens d'aliéné. L'identité objective (le point fixe) demeure. L'importance accordée à la mémoire par l'histoire des Amériques amérindienne et blanche le rappelle. L'enjeu du roman n'est pas de la nier, mais de l'explorer, comme une interférence obligée, si ce mot peut se voir attribuer une valeur à la fois négative et positive. La «bibliothèque» historique (la mémoire collective) fait partie du minibus où qu'il aille. Au terme de son aventure, Jack revient à Montréal, ce qui est, sans l'être tout à fait, son point d'ancrage (Québec), débarrassé de quelques images et d'une carapace devenue encombrante (le Volks). La 
lumière qu'il laisse toujours allumée, même en voyage, dans son appartement de Québec, montre que le voyage n'est pas une fuite en avant, mais bien une forme d'exploration (p. 289).

Ce même Volks, en sa qualité de «maison/mobile» est la figure emblématique du roman et justifie pleinement sa présence dans le titre. Comme véhicule, il appartient au mouvement: il déplace vers un ailleurs. Comme habitacle, il appartient au fixe: il garde chez soi. L'identité, la culture, l'écriture ne sont-ils pas mixtes comme cette «maison mobile» qui semble tout à fait convenir à la réalité mixte de Pitsémine/la Grande Sauterelle qui agit dans le roman comme pôle d'attraction:

Elle avait une grande affection pour le Volks, même s'il était vieux et lent et capricieux. [...] C'était comme une maison... La fille n'avait jamais eu de vraie maison. Elle était venue au monde dans une roulotte parce que sa mère, en épousant un Blanc, avait perdu la maison qu'elle possédait sur la réserve de La Romaine; elle avait été expulsée et elle avait perdu son statut d'Indienne. Mais les Blancs, de leur côté, la considéraient toujours comme une Indienne et ils avaient refusé de louer ou de vendre une maison aux nouveaux mariés. Finalement, ses parents avaient acheté une roulotte. (p. 99)

On peut enfin supposer que ce Volks, qui est très vieux, qui a appartenu à plusieurs propriétaires, qui a été acheté en Allemagne, a parcouru l'Europe, traversé l'océan, a fait la côte Est de l'Amérique du Nord et finalement entrepris le voyage vers l'Ouest, a traversé le temps québécois, de la Révolution tranquille jusqu'au début des années 80 , et gardé des traces (collants, coquillages, cartes routières) des événements, des luttes gagnées ou perdues, des mouvements politiques, idéologiques, culturels, etc. qui ont jalonné sa route. Mais le propre d'un Volks, même vieux, c'est d'être increvable, de rouler toujours, non de s'arrêter. Ainsi en est-il du texte de Poulin qui avance sans se nier ou s'arrêter sur une forme donnée, qui traverse et enregistre les discours, les langages, les images québécoises, sans pourtant s'enfermer dans une formule figée comme à l'intérieur de frontières qu'on aurait imaginées, à la suite d'un traumatisme (celui du référendum, par exemple?), trop étanches pour être traversées. Volkswagen Blues, et c'est de cette manière qu'il prend sa place dans le texte québécois, résiste à la réduction des discours en explorant la culture comme une unité ouverte, en devenir, ni déterminée ni prédéterminée, capable de se perdre ou de se renouveler, se transcendant elle-même ${ }^{30}$. Le roman de Poulin se rattache à une écriture pour qui «l'indépendance est déjà commencée», cette expression étant à prendre dans le sens que lui donne Belleau: Tout ce qui chez nous tend à diversifier, complexifier, étendre et renouveler le champ des discours travaille, en fin de compte, pour l'indépendance. ${ }^{31}$ Par là il est à ranger à côté, et à lire avec d'autres textes, notamment ceux appartenant à l'écriture immigrante, qui,

30 Mikhail Bakhtine, op. cit., p. 354.

31 André Belleau, op. cit., p. 139. 
comme celui de Poulin, ne sont possibles que dans la perspective d'une indépendance déjà commencée, entrevue moins comme une fin à atteindre absolument que comme un espace nécessairement pluritonal à aménager ${ }^{32}$.

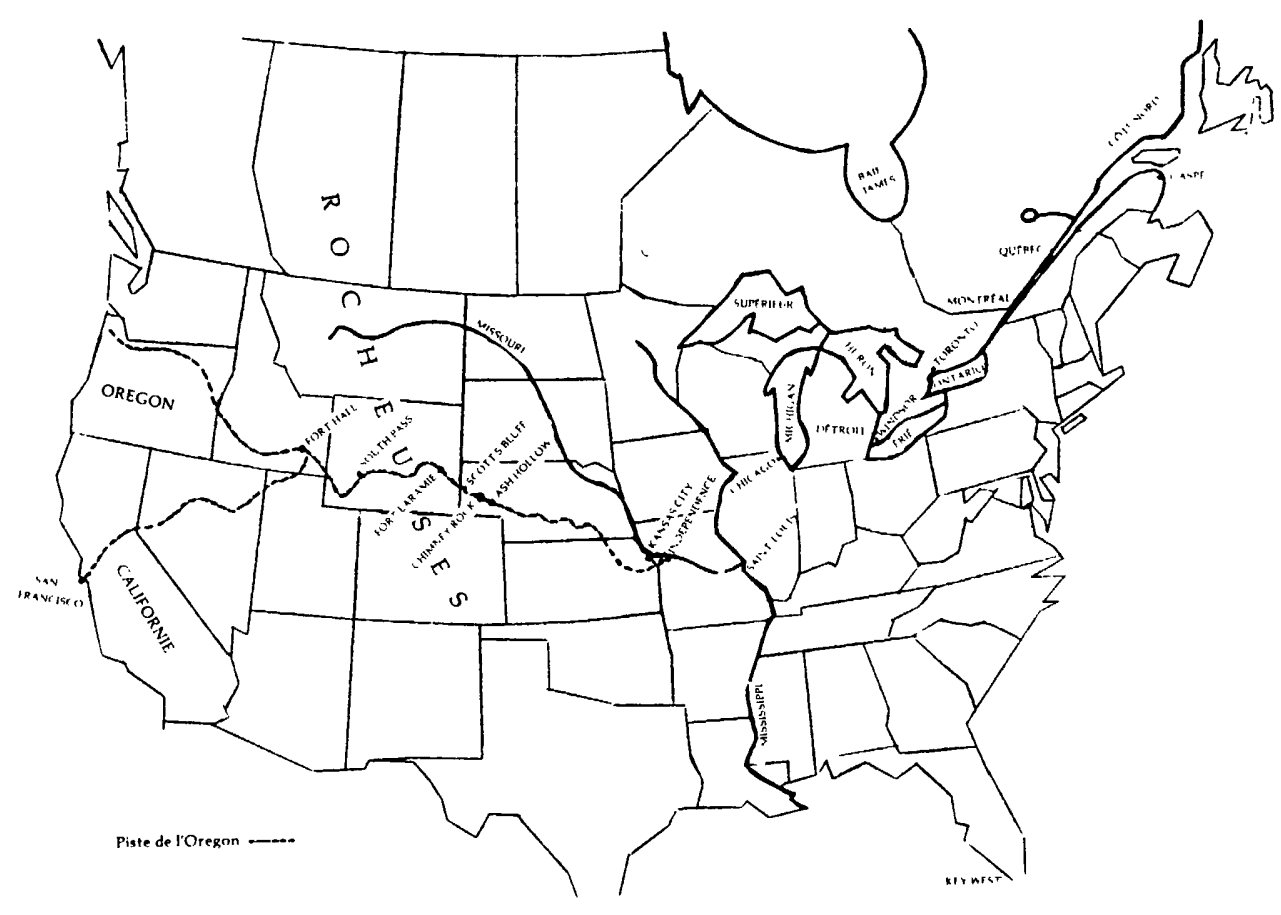

32 Le «Lecteur modèle», tel que le définit Umberto Eco (Lector in fabula), postulé par la stratégie textuelle, peut donc ici être assez divers et nombreux. Ce peut être celle ou celui qui a roulé en Volks (minibus ou coccinelle), qui s'approche donc de l'âge de Jack et de celui de l'auteur, comme ce peut être aussi celle ou celui qui un peu plus tard roulera en Renault 5, ou celui qui, beaucoup plus jeune, doit se satisfaire du vélo... Ce peut être la Québécoise ou le Québécois de «vieille souche» ou la Québécoise ou le Québécois appartenant à la communauté immigrante ou à la minorité anglaise. En fait, ne serait-ce pas tout simplement la Québécoise ou le Québécois d'aujourd'hui, à vingt, trente, quarante, cinquante ans..., né à Chicoutimi, à Montréal, dans le West Island, à Québec, au Viêt-nam, à Vancouver, en Italie, au Mexique, au Liban... Chacun et chacune peut sans doute trouver dans cet espace poreux, l'espace de sa liberté... Cela, toutefois, reste à vérifier. 